\title{
Study of cubic and hexagonal cell geometries of a 3D diamond detector with a proton micro-beam
}

DOI:

10.1016/j.diamond.2017.06.014

10.1016/j.diamond.2017.06.014

\section{Document Version}

Accepted author manuscript

Link to publication record in Manchester Research Explorer

\section{Citation for published version (APA):}

Booth, M. J., Forcolin, G. T., Grilj, V., Hamilton, B., Haughton, I., McGowan, M., Murphy, S. A., Oh, A., Salter, P. S., Sudi, I., \& Skukan, N. (2017). Study of cubic and hexagonal cell geometries of a 3D diamond detector with a proton micro-beam. Diamond and Related Materials. https://doi.org/10.1016/j.diamond.2017.06.014, https://doi.org/10.1016/j.diamond.2017.06.014

\section{Published in:}

Diamond and Related Materials

\section{Citing this paper}

Please note that where the full-text provided on Manchester Research Explorer is the Author Accepted Manuscript or Proof version this may differ from the final Published version. If citing, it is advised that you check and use the publisher's definitive version.

\section{General rights}

Copyright and moral rights for the publications made accessible in the Research Explorer are retained by the authors and/or other copyright owners and it is a condition of accessing publications that users recognise and abide by the legal requirements associated with these rights.

\section{Takedown policy}

If you believe that this document breaches copyright please refer to the University of Manchester's Takedown Procedures [http://man.ac.uk/04Y6Bo] or contact uml.scholarlycommunications@manchester.ac.uk providing relevant details, so we can investigate your claim.

\section{OPEN ACCESS}




\section{Accepted Manuscript}

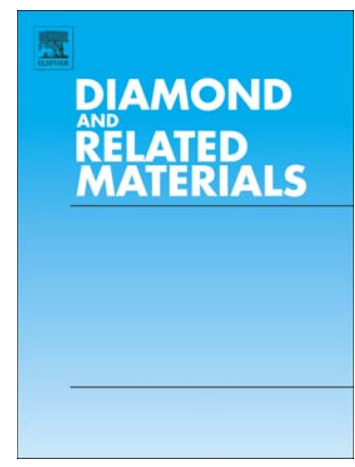

Study of cubic and hexagonal cell geometries of a 3D diamond detector with a proton micro-beam

M.J. Booth, G.T. Forcolin, V. Grilj, B. Hamilton, I. Haughton, M. McGowan, S.A. Murphy, A. Oh, P.S. Salter, I. Sudić, N. Skukan

PII:

S0925-9635(17)30193-0

DOI: $\quad$ doi: $10.1016 /$ j.diamond.2017.06.014

Reference: $\quad$ DIAMAT 6901

To appear in: $\quad$ Diamond \& Related Materials

Received date: $\quad 11$ April 2017

Revised date: $\quad 25$ June 2017

Accepted date: $\quad 26$ June 2017

Please cite this article as: M.J. Booth, G.T. Forcolin, V. Grilj, B. Hamilton, I. Haughton, M. McGowan, S.A. Murphy, A. Oh, P.S. Salter, I. Sudić, N. Skukan, Study of cubic and hexagonal cell geometries of a 3D diamond detector with a proton micro-beam, Diamond \& Related Materials (2017), doi:10.1016/j.diamond.2017.06.014

This is a PDF file of an unedited manuscript that has been accepted for publication. As a service to our customers we are providing this early version of the manuscript. The manuscript will undergo copyediting, typesetting, and review of the resulting proof before it is published in its final form. Please note that during the production process errors may be discovered which could affect the content, and all legal disclaimers that apply to the journal pertain. 
Study of cubic and hexagonal cell geometries of a 3D diamond detector with a proton micro-beam

- April $2017 \bullet$ Diamond and Related Materials

Study of cubic and hexagonal cell
geometries of a 3D diamond detector
with a proton micro-beam

\author{
M. J. Booth ${ }^{1}$, G. T. Forcolin'², V. Grilj ${ }^{3}$, B. Hamilton ${ }^{4}$, I. Haughton ${ }^{2}$, M. McGowan ${ }^{4}$,

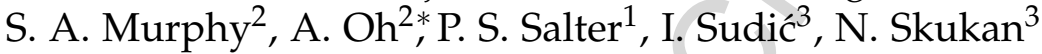 \\ 1. Department of Engineering Science, University of Oxford, UK \\ 2. School of Physics and Astronomy, University of Manchester, UK \\ 3. Ruđer Bošković Institute, Zagreb, Croatia \\ 4. School of Electrical and Electronic Engineering, University of Manchester, UK
}

\begin{abstract}
A detector from single crystal synthetic diamond with conducting wires has been prepared with an improved femto-second laser process. The detector was characterised with a $4.5 \mathrm{MeV}$ proton micro-beam (Ruđer Bošković Institute, Zagreb). The charge collection efficiency and the transient current response have been investigated with high spatial resolution. A hexagonal and square cell geometry is investigated. Both cell geometries show full charge collection at $40 \mathrm{~V}$ bias voltage, and little charge sharing between neighbouring cells. The experimental data is compared to a simulation and qualitative agreement is observed.
\end{abstract}

\section{INTRODUCTION}

Diamond detectors with conductive wires in the bulk, so called 3D diamond detectors, have been demonstrated to work as position sensitive, segmented detectors [1], and to exhibit superior radiation hardness compared to conventional planar diamond detectors [2]. In this study we examine the charge collection properties of a novel generation of 3D diamond detectors fabricated with a femto-second laser and aberration correcting optics.

The paper is structured as follows: Section 2 describes the production process of the 3D diamond test detector. Section 3 presents studies of the charge collection properties with a proton micro-beam including spatially resolved transient currents and a comparison of the experimental data to simulation results.

\section{Detector FABRicAtion}

To trace out conducting wires in diamond a sufficiently large photon density is needed to achieve non-linear absorption of photons and in consequence localised phase transition of diamond into a combination of a graphitic phase and amorphous carbon [3].

The conductive wires in the test device are fabricated with a short laser pulse of $100 \mathrm{fs}$ duration at a wavelength of $790 \mathrm{~nm}$ and a repetition rate of $1 \mathrm{kHz}$ from an amplified Ti:sapphire laser (SpectraPhysics Solstice), operating in $\mathrm{TEM}_{00}$ beam mode [4]. Focussing the laser inside the diamond, there is severe spherical aberration due to the refractive index mismatch at the diamond interface, limiting the efficiency of the laser fabrication. To counter this a liquid crystal spatial light modulator (SLM) was employed to adaptively correct for the aberration during fabrication [5], ensuring that a well defined diffraction limited focal spot is achieved inside the diamond. Using

\footnotetext{
${ }^{*}$ Corresponding author: alexander.oh@manchester.ac.uk
} 
aberration correction in the fabrication gives a better yield and improves the wire properties significantly compared to conventional processing [6].

The diamond sample was mounted to a programmable 3-axis stage to control the location of the focal point of the beam within the diamond sample with a fixed translational speed of $10 \mu \mathrm{m} / \mathrm{s}$. The laser power was adjusted using an adjustable half wave plate in conjunction with a polarizer, and the laser beam was focused to a diameter of $\leq 1 \mu \mathrm{m}$ using an air based objective lens with numerical aperture 0.75 .

A single crystal electronic-grade sample obtained from IIa[7], with dimensions $4.0 \times 4.0 \times$ $0.4 \mathrm{~mm}^{3}$ was used as substrate. An array of graphitic wires was written into the diamond, arranged such that square, rectangular and hexagonal shaped detector cells were formed. The wires have a diameter of about $2 \mu \mathrm{m}$.

A structured metallisation pattern was applied to the front and back side of the sample to provide electrical contacts to the conductive wires. The metallisation was achieved with an evaporation process and consists of a chromium layer of $50 \mathrm{~nm}$ thickness providing adhesion to the diamond, and a gold layer of $80 \mathrm{~nm}$ thickness to prevent oxidization of the chromium layer. The sample was annealed at $400{ }^{\circ} \mathrm{C}$ for 4 minutes in a nitrogen atmosphere.

Three different detector structures have been placed on the sample as shown in Figure 1a: a planar metal strip array for reference; a 3D array consisting of square cells, rectangular cells, and hexagonal cells (Figure 1b) all with $100 \mu \mathrm{m}$ pitch; and a 3D "phantom" array, i.e. an area where the same metallization pattern used to connect the graphitic wires is applied but without wires, for comparing the charge collected by the metallization alone. Sets of readout electrodes of the 3D structures were ganged together to form strips. The bias electrodes were connected from the opposite side to a common biasing pad. The electrodes exhibit an ohmic behavior until approximately $\pm 40 \mathrm{~V}$, and show an exponential increase in current for higher voltages. The resistivity has been determined to $0.7 \pm 0.1 \Omega \mathrm{cm}$.

The structure of the electrode at the surface was imaged using a Scanning Electron Microscope (SEM) and compared to structures which were fabricated without adaptive optical correction of the spherical aberration. Figure 2 shows both samples exhibit at the end of the conductive wires small craters on the surface where the laser exited the diamond during the processing. The lower density of the electrode material compared to the diamond causes pressure inside the diamond sample and is sufficiently high to eject a few microns of diamond material at the surface. The two images reveal that aberration correction during fabrication allows a better control on the electrode diameter. A periodic surface structuring can be seen in Figure $2 \mathrm{a}$, which is characteristic of the interaction between a linearly polarized ultrafast laser and the diamond surface [8].

\section{Proton test beAm}

A proton micro-beam [9] at the Ruđer Bošković Institute in Zagreb was used to study the detector by ion beam induced charge (IBIC) and time resolved ion beam induced current (TRIBIC) [10]. It is based on a Van-de-Graaff tandem accelerator that can accelerate protons to an energy of $4.5 \mathrm{MeV}$ and produce a microbeam with an FWHM of $\leq 2 \mu \mathrm{m}$. The proton stopping range in diamond is approximately $97 \mu \mathrm{m}$ at $4.5 \mathrm{MeV}[11]$, thus protons will stop within the detector volume. The spatial distribution of the ionisation charge is estimated using SRIM [11], a software package to simulate ion transport in matter. The FWHM of the charge distribution in the transverse plane is estimated to be less then $5 \mu \mathrm{m}$ at the stopping distance.

The sample was mounted on a PCB specifically designed for optimised high frequency response. A single strip was wire bonded to a read-out pad (channel 1). The adjacent two strips on either side of the first strip were ganged together and connected to a second read-out pad (channel 2). The bias voltage was connected to the entire detector. The signal lines are connected via SMA connectors to the read-out electronics. For the IBIC measurements the read-out chain consisted of a charge integrating amplifier followed by a shaping 
Study of cubic and hexagonal cell geometries of a 3D diamond detector with a proton micro-beam - April $2017 \bullet$ Diamond and Related Materials

amplifier with a shaping time of $2 \mu \mathrm{s}$. A selftriggered data acquisition system recorded the amplitude of the charge signal with a trigger threshold of 20ke, as well as the position of the proton beam. For the TRIBIC measurements the read-out chain was modified to record transient currents. The current signal generated by the $4.5 \mathrm{MeV}$ proton was amplified with a Cividec C2 2 GHz broadband current amplifier [12] and digitised with a WaveMaster-8500 $5 \mathrm{GHz}$ sampling scope from Lecroy. The waveform and the position of the proton beam was recorded with a self-triggered data acquisition system. The read-out chains were calibrated with a silicon detector assuming an ionisation energy of $3.6 \mathrm{eV}$ per electron-hole pair in silicon.

\subsection{IBIC Results}

The diamond test detector has been charaterised in terms of a charge collection efficiency (CCE), defined as the ratio of measured amount of charge from the detector, and the produced amount of charge in the diamond by the ionizing proton. The CCE has been measured as a function of position on the detector with a spatial resolution of a few microns.

The results on the CCE measured on square and hexagonal shaped cell with the proton micro-beam are shown in Figure 3. The data obtained with the read-out channel 1 are shown. The shape of the hexagonal and square cells is clearly visible. The conducting wires in the diamond bulk exhibit a lower CCE and show as darker areas. Data taken at a bias voltage of $U_{b}=-2 \mathrm{~V}$ show a less sharp boundary between channel 1 and the cells connected to channel 2, while the separation is well defined for $U_{b}=-20 \mathrm{~V}$ and the shape of the cells is rendered clearly. With increasing applied bias voltage the CCE both increases and becomes more uniform across the cell, as can be seen in comparing Figures $3 b$ and $3 d$ to $3 a$ and $3 c$.

The CCE averaged over data from channel 1 and 2 are shown in Figure 4 as a function of bias voltage, $U_{b}$. No data was recorded in the square array at $+40 \mathrm{~V}$ due to high leakage current. The measured CCE is consistent for both square and hexagonal cells and symmetric for positive and negative bias voltages, reaching close to full charge collection at $\left|U_{b}\right| \geq 20 \mathrm{~V}$.

The charge deposited in the detector by the incident proton can be shared by adjacent cells if the proton impacts in the region of the cell boundary. This 'charge sharing' and can be parameterised as follows:

$$
\eta=\frac{q_{2}}{q_{1}+q_{2}}
$$

where $\eta$ is the fraction of the total charge collected by the outer channel 2 , and $q_{1}$ and $q_{2}$ the values of charge collected with channels 1 and 2, respectively. Figure 5 shows the value of $\eta$ as measured in the square and hexagonal cells at $2 \mathrm{~V}$ and $20 \mathrm{~V}$. In averaging over several horizontal slices, a charge sharing width was calculated by taking the width of the $25 \%$ to $75 \%$ transition of the $\eta$ value in the cell boundary region. In the case of the hexagonal cell, the tilt of the cell edge was applied before measuring the width. The $\eta$ transition region at the left and right of the central cell was averaged. The variation of the charge sharing width as a function of applied bias is shown in Figure 6. The observed charge sharing $\eta$ is about $25 \%$ lower for the hexagonal cells compared to the square cells for the same value of $U_{b}$. The covered area of a single hexagonal and square cell are comparable, $11500 \mu \mathrm{m}^{2}$ and $10000 \mu^{2}$, respectively, with the hexagonal cell covering a $15 \%$ larger area. The charge sharing region for $U_{b} \geq-40 \mathrm{~V}$ is $15 \mu \mathrm{m}$ for hexagonal and $22 \mu \mathrm{m}$ for cubic cell geometry.

The strong dependence of the charge sharing width on the bias voltage is likely due to the longer drift time and thus higher diffusion of the initial charge cloud at low fields. Additionally, the charge collection efficiency is lower $\left(\approx 90 \%\right.$ at $\left.U_{b}=5 \mathrm{~V}\right)$ at low fields as shown in Figure 4 . The trapped fraction of the charge can induce partly on the neighboring electrodes, as reported in [1], leading to a widening of the $\eta$ distribution.

\subsection{TRIBIC Measurements and Simu- lation}

TRIBIC allows to investigate the electric field distribution inside the 3D diamond detector. The transient current signal (TCT) is recorded along with the impact position of a 
single proton. The analysis of the TCT signal allows to reconstruct the electric field distribution. For a planar electrode configuration the electric field distribution does not vary with the proton impact position and the TCT signal will thus not vary for a perfect detector. In the case of the 3D electrode structure in the square and hexagonal cells, the electric field distribution is non-uniform and the transient current signal shape is expected to vary as a function of the impact position of the proton in the cell. The complex field structure and resulting TCT signal is simulated and compared to the measurements.

\subsubsection{Measurements}

Measurements were made on hexagonal and square cells at $U_{b}=20 \mathrm{~V}$ for positive and negative polarity, with the proton beam incident on the read-out side. A square cell was also investigated at the bias side. Figures $7 \mathrm{a}$ and $7 \mathrm{~b}$ show a comparison of the averaged TRIBIC signal for both square and hexagonal cells at two different field regions within the cells, a high and low field region, as indicated on the plot, on the read-out side. The amplitudes observed are summarised in Table 1.

The amplitude values of the current pulses are about a factor two smaller for low field regions compared to high field regions. The hexagonal cell type has slightly higher amplitude for both regions compared to the cubic cell. These observation can be explained by the field shape and are reproduced by a simulation model explained below. The cumulative integral of the current pulses are shown in Figures $7 c$ and $7 d$, the lower amplitude of the the square cell results in a slower rise of the integral compared to the hexagonal cell. The visualization of the maximum amplitude of the current pulses as a function of the incident position of the proton in Figure 8 reveals the variation is related to the electrode geometry. In this figure the cell boundary is indicated by solid line, the electrodes by the open circles, and additionally the low field (open crosses) and high field (open stars) regions used for Figure 7 are indicated. The collection time of the charge signal is strongly dependent on the position and follows the electric field distribution.
A four-fold and six-fold symmetry given by the electric field geometry is clearly visible for the cubic and hexagonal cell structure, respectively. The larges pulses are observed in the vicinity of the central electrode. The pattern for positive and negative polarity show a slight variation, likely due to the difference in electron and hole mobility. A simulation study (see Section 3.2.2) could qualitatively reproduce the symmetry, but not the exact shape of the distribution.

TRIBIC measurements were also taken on the bias side of the detector. Figures 9a and $9 \mathrm{~b}$ show a density plot of the recorded current traces on the readout and bias sides of the detector, respectively. The pulse shapes vary considerably on the readout side of the detector as expected from the amplitude distributions discussed in the previous paragraph. For the bias side the variation is much smaller and a dominant pulse shape is visible, with a FWHM of $\approx 35 \mathrm{~ns}$. The lack of structure on the bias side is likely due to the effectively increased RC constant seen by the induced charge. The effective electrode resistance experienced by the incident protons is increased for the bias side compared to the read-out side, since at the read-out side the effective electrode length is approximately equal to the penetration depth of the proton, i.e. $100 \mu \mathrm{m}$. When the proton is incident on the bias side the electrode length for the induced charge is the difference between detector thickness and penetration depth, i.e. three times larger with approximately $300 \mu \mathrm{m}$. A similar consideration applies to the effective capacitance, resulting in an increased RC constant for protons incident on the bias side. The $\mathrm{RC}$ constant dominates the transient current signal for the bias side, and thus the amplitude and FWHM of the current signal is independent of the position in the cell as seen in Figures $9 \mathrm{c}$ and $9 \mathrm{~d}$. From the measurement of the capacitance and resistance the $\mathrm{RC}$ constants was estimated to $\approx 30$ ns for the bias side measurement which corroborates the observation of no visible significant structure.

\subsubsection{Simulation}

Simulations were carried out using Synopsys TCAD[13] in two (2D) and three spacial dimensions (3D) to understand the observed 
Study of cubic and hexagonal cell geometries of a 3D diamond detector with a proton micro-beam - April 2017 • Diamond and Related Materials

\begin{tabular}{|c|c|c|c|} 
Field & $U_{b}[\mathrm{~V}]$ & Cell type & Amplitude $[\mu \mathrm{A}]$ \\
\hline Low & +20 & Hexagonal & $10.9 \pm 0.8$ \\
Low & +20 & Square & $9.4 \pm 0.9$ \\
Low & -20 & Hexagonal & $12.3 \pm 0.7$ \\
Low & -20 & Square & $11.8 \pm 0.9$ \\
High & +20 & Hexagonal & $25.2 \pm 1.4$ \\
High & +20 & Square & $21.2 \pm 0.5$ \\
High & -20 & Hexagonal & $21.0 \pm 1.2$ \\
High & -20 & Square & $19.0 \pm 0.8$
\end{tabular}

Table 1: Summary of the amplitude values for TRIBIC measurements at different field values and cell configurations.

behavior. Simulations in three spatial dimensions are very CPU intensive and could be run only for a few events. Simulations in two spatial dimensions (2D) are deployed for predictions on a larger area of the detector. For the $2 \mathrm{D}$ simulation a mesh representing an area of $2 \times 2$ square cells was created to include the effects of neighboring cells. Due to the 2D nature of the simulation it is not possible to accurately model a Bragg Peak, however a selection of hits were compared to simulations using a 3D quarter cell mesh to validate that the $2 \mathrm{D}$ results are a good approximation to what is obtained with a full 3D simulation.

The 3D simulations were performed using a column resistance of $175 \mathrm{k} \Omega$ and a simulated charge density of $5.882 \times 10^{-5} \mathrm{pC} \mathrm{m}^{-1}$ from the diamond surface to a depth of $100 \mu \mathrm{m}$, and

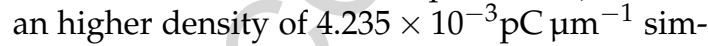
ulated at a depth between $95-105 \mu \mathrm{m}$ to approximate a Bragg peak.

For the 2D simulations the $\mathrm{RC}$ constant of the 3D device was adjusted by increasing the resistance for the electrodes, and the simulated charge density was set to $6.4 \times 10^{-6} \mathrm{pC}$.

A bandgap energy of $5.5 \mathrm{eV}$ and a dielectric constant of 5.7 were used in the simulation[14]. The simulation input parameters for the modelling of the carrier dynamics were for the electron saturation velocity $v_{s, e}=9.6 \times 10^{6} \mathrm{~cm} \mathrm{~s}^{-1}$; hole saturation velocity $v_{s, h}=14.1 \times 10^{6} \mathrm{~cm} \mathrm{~s}^{-1}$; low field electron mobility $\mu_{0, e}=1714 \mathrm{~cm}^{2} \mathrm{~V}^{-1} \mathrm{~s}^{-1}$; and low field hole mobility $\mu_{0, h}=2064 \mathrm{~cm}^{2} \mathrm{~V}^{-1} \mathrm{~s}^{-1}$ [15]. These values are used in a Caughey-Thomas model [16] to calculate the field dependent mobility. To simulate the effect of the amplifier chain, the signal frequency spectrum was limited to $2 \mathrm{GHz}$ corresponding to the bandwidth limit of the current amplifier.

Figure 10 compares simulation and data for the hexagonal and cubic cells in the low high field regions. Shown are the results for a bias voltage of $20 \mathrm{~V}$ and negative polarity. The agreement of data and simulation is reasonable taking into account the simplification of deploying an effective resistance and the amplifier model. The basic features differentiating the low and high field regions are qualitatively reproduced. The slower turn on of the low field region is visible for both hexagonal and cubic cells. A distinct two peak structure caused by the different time structure of the signal contribution from holes and electrons is resolved in the hexagonal cell low field case in both the simulation and measurement, however it is less pronounced in the case of the simulation.

Figure 11 shows a comparison of the amplitude as a function of position on both hexagonal and cubic cell types. The hexagonal and cubic symmetries are visible, related to the electric field distribution. The amplitude range is comparable to the measurement data shown in Figure 8a and Figure 8c for the hexagonal and cubic cell, respectively. The patterns seen in the amplitude distribution in data and simulation show some differences. The sixfold and fourfold symmetries for the hexagonal and cubic cell are clearly visible, however the shape is not accurately reproduced by the simulation while the amplitude range is comparable. 
Study of cubic and hexagonal cell geometries of a 3D diamond detector with a proton micro-beam - April 2017 • Diamond and Related Materials

\section{Conclusions}

A 3D diamond detector with square and hexagonal cell geometry was produced with an improved graphitisation process using spherical aberration corrected laser optics. The detector response to a proton micro-beam was studied. The response to the proton beam is consistent with full charge collection within the active regions of the detector. Significant charge sharing between neighbouring cells has been observed for low bias voltage. Both square and hexagonal cells show full charge collection at a bias voltage of $40 \mathrm{~V}$, however the hexagonal geometry shows about $25 \%$ less charge sharing than the square cells. The charge sharing properties are important for charged particle tracking applications, where sharing of charge is beneficial to improve the track resolution, and for dosimetry applications, where a defined active volume is important and charge sharing is disadvantageous.

With the TRIBIC (time resolved ion beam induced current) technique the time profile of the induced currents were studied as a function of position and bias voltage. The experimental data have been compared to simulations. A significant dependence of the transient current shape on the location in the cell due to the electric field distribution has been observed experimentally and is reproduced with a simulation. These studies have been performed on an un-irradiated diamond sample. For both medical dosimetry and particle tracking applications the influence of radiation damage on the charge collection properties for different cell geometries are important, and will be investigated as the next step.

\section{ACKNOWLEDGEMENTS}

AO would like to acknowledge support from the Royal Society through grant UF120106 and STFC grant ST/M003965/1/. MJB and PSS were supported through a Leverhulme Trust grant (RPG-2013-044). The measurements at RBI were supported by the H2020 project AIDA-2020, GA no. 654168. We thank the RD42 collaboration for fruitful discussions.

\section{REFERENCES}

[1] F. Bachmair, L. Bä, P. Bergonzo, B. Caylar, G. Forcolin, I. Haughton, D. Hits, H. Kagan, R. Kass, L. Li, A. Oh, S. Phan, M. Pomorski, D. S. Smith, V. Tyzhnevyi, R. Wallny, and D. Whitehead, "A 3D diamond detector for particle tracking," NIM Phys A 786, 2015.

[2] S. Lagomarsino, M. Bellini, C. Corsi, V. Cindro, K. Kanxheri, A. Morozzi, D. Passeri, L. Servoli, C. J. Schmidt, and S. Sciortino, "Radiation hardness of threedimensional polycrystalline diamond detectors," Applied Physics Letters 106, 2015.

[3] T. V. Kononenko, M. Meier, M. S. Komlenok, S. M. Pimenov, V. Romano, V. P. Pashinin, and V. I. Konov, "Microstructuring of diamond bulk by IR femtosecond laser pulses," Applied Physics A 90, 2008.

[4] Newport Corporation, "Spectra Physics Solstice Data Sheet." https://assets. newport.com/webDocuments-EN/images/ Solstice_Ultrafast_Laser_SP.pdf, 2013. [Accessed 07-Nov-2016].

[5] B. Sun, P. S. Salter, and M. J. Booth, "High conductivity micro-wires in diamond following arbitrary paths," Applied Physics Letters, vol. 105, 2014.

[6] S. Murphy, M. Booth, L. Li, A. Oh, P. Salter, B. Sun, D. Whitehead, and A. Zadoroshnyj, "Laser processing in $3 \mathrm{~d}$ diamond detectors," Nuclear Instruments and Methods in Physics Research Section A: Accelerators, Spectrometers, Detectors and Associated Equipment, vol. 845, pp. 136 - 138, 2017. Proceedings of the Vienna Conference on Instrumentation 2016.

[7] IIa Technologies Pte. Ltd, "IIa Diamond Properties." http://2atechnologies. com/2a-diamond-properties/, 2013. [Accessed 08-Nov-2016].

[8] M. Shinoda, R. R. Gattass, and E. Mazur, "Femtosecond laser-induced formation of nanometer-width grooves on synthetic single-crystal diamond surfaces," Journal 
Study of cubic and hexagonal cell geometries of a 3D diamond detector with a proton micro-beam - April 2017 • Diamond and Related Materials

of Applied Physics, vol. 105, no. 5, p. 053102, 2009.

[9] M. Jakšić, I. Bogdanović Radović, M. Bogovac, V. Desnica, S. Fazinić, M. Karlušić, Z. Medunić, H. Muto, Z̆. Pastuović, Z. Siketić, N. Skukan, and T. Tadić, "New capabilities of the Zagreb ion microbeam system," NIM Phys B, 260, 2007.

[10] M. Breese, E. Vittone, G. Vizkelethy, and P. Sellin, "A review of ion beam induced charge microscopy," Nuclear Instruments and Methods in Physics Research Section B: Beam Interactions with Materials and Atoms, vol. 264, no. 2, pp. $345-360,2007$.

[11] J. Ziegler, "Stopping and Range of Ions in Matter." http://www.srim.org/, 2013. [Accessed 01-Nov-2016].

[12] CIVIDEC Instrumentation, "Current Amplifier Data Sheet." https ://cividec.at/ files/15_pdf.pdf, 2015. [Accessed 01Nov-2015].

[13] Synopsys Inc., "Synopsys TCAD." http: //www.synopsys.com/home.aspx/, 2015. [Accessed 28-April-2015].

[14] L. S. Pan, S. Han, and D. R. Kania, Diamond: Electronic Properties and Applications. Kluwer Academic, Dordrecht, 1995.

[15] H. Pernegger, S. Roe, P. Weilhammer, V. Eremin, H. Frais Kölbl, E. Griesmayer, H. Kagan, S. Schnetzer, R. Stone, W. Trischuk, D. Twitchen, and A. Whitehead, "Charge-carrier properties in synthetic single-crystal diamond measured with the transient-current technique," $A p$ plied Physics 97, 2005.

[16] D. M. Caughey and R. E. Thomas, "Carrier mobilities in silicon empirically related to doping and field," in Proc. IEEE vol 52 (IEEE, ed.), 1967. 
Study of cubic and hexagonal cell geometries of a 3D diamond detector with a proton micro-beam - April 2017 • Diamond and Related Materials
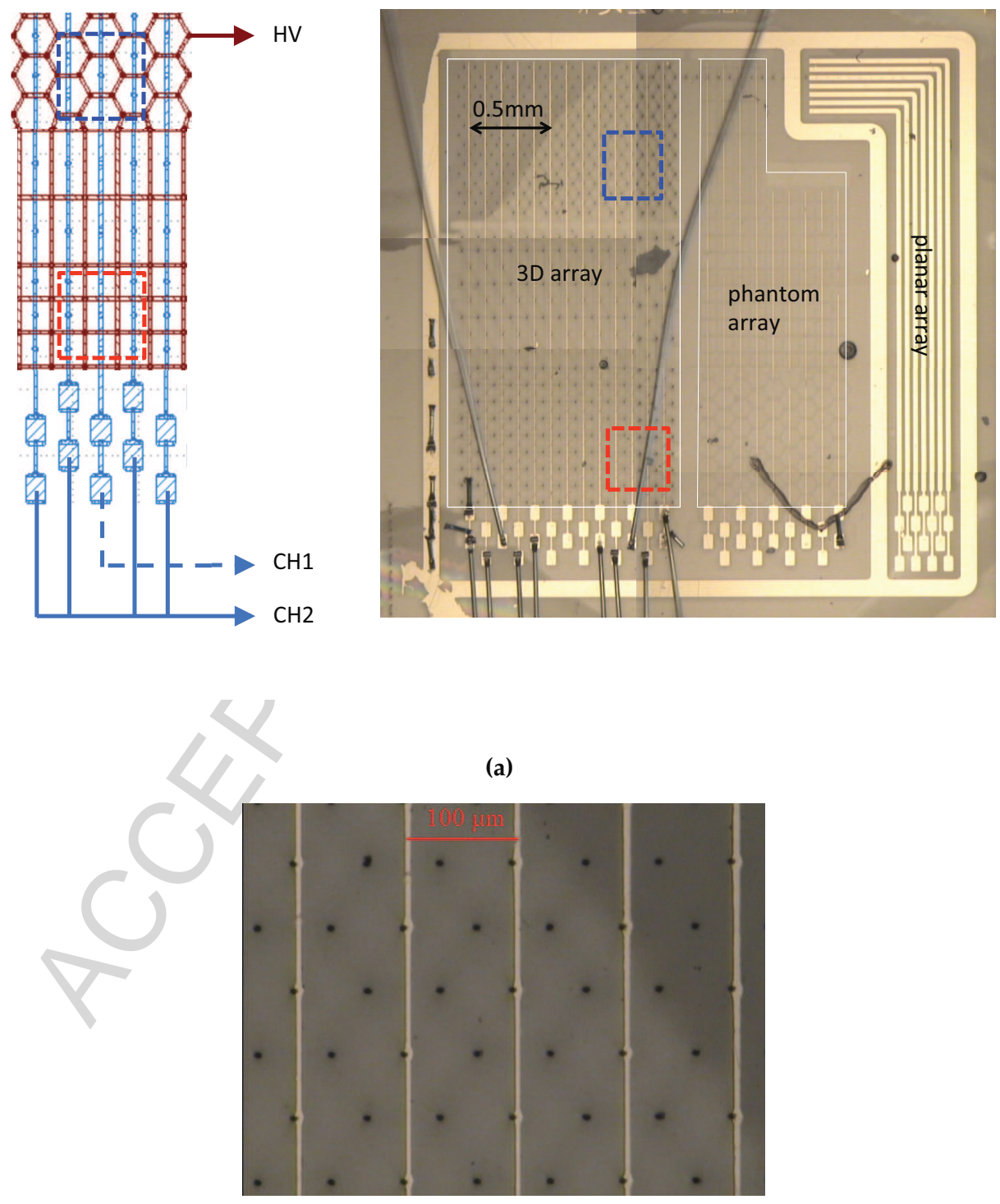

(b)

Figure 1: Optical images of the detector. The metallisation and readout strips are shown in gold. (a) the three areas are: Left, planar array; Middle, 3D phantom array; Right, 3D array. The detector connection scheme (not to scale) with bias voltage (HV) contacted to the back side, and channel 1 (CH1) and channel 2 (CH2) connected to the current amplifiers is shown. The areas enclosed in the dashed line correspond to the measurement locations. (b) hexagonal cell array, with the readout strips in gold and shadows of the bias connections for the hexagonal cells also visible.) 
Study of cubic and hexagonal cell geometries of a 3D diamond detector with a proton micro-beam - April $2017 \bullet$ Diamond and Related Materials

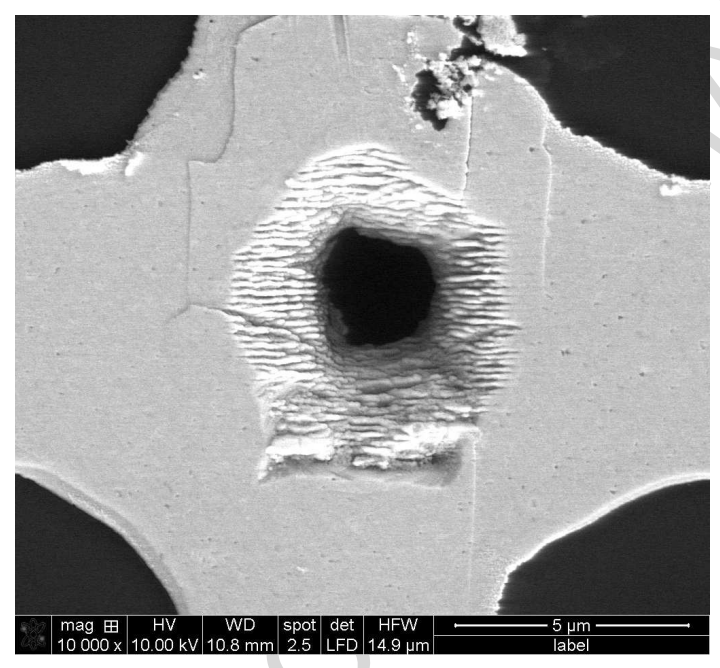

(a)

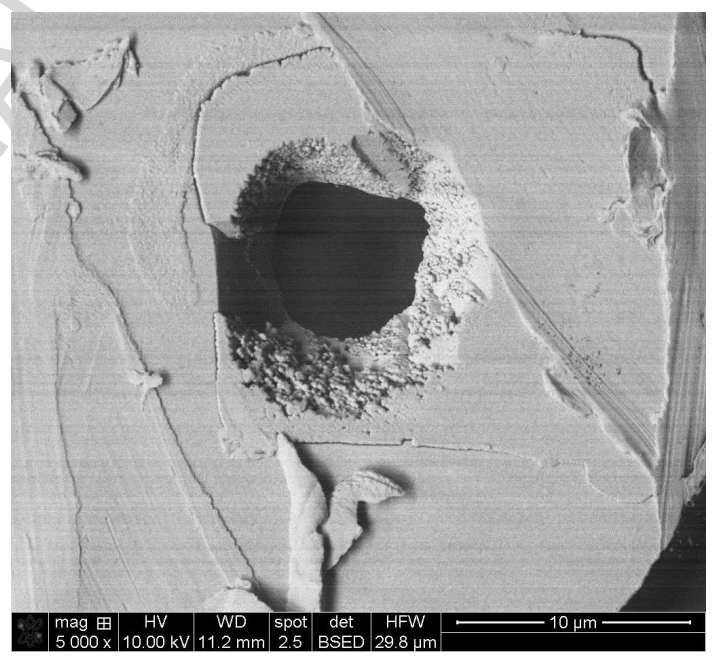

(b)

Figure 2: A comparison of SEM images for (a) a sample produced with optical aberration correction and (b) a sample produced without correction. The scale of the SEM in (a) (15 $\mu \mathrm{m}$ width) is smaller by a factor two compared to (b) (30 $\mu \mathrm{m}$ width). The electrode diameter in (a) is significantly smaller due to greater control in the electrode formation. 
Study of cubic and hexagonal cell geometries of a 3D diamond detector with a proton micro-beam - April 2017 • Diamond and Related Materials

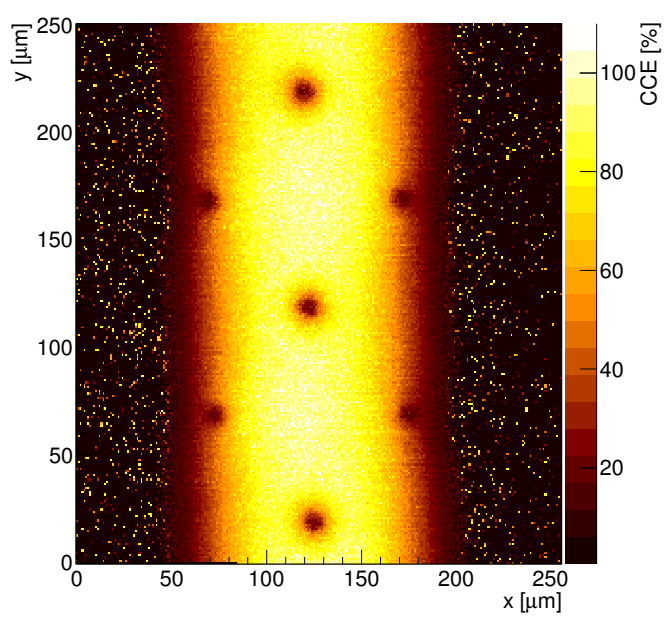

(a)

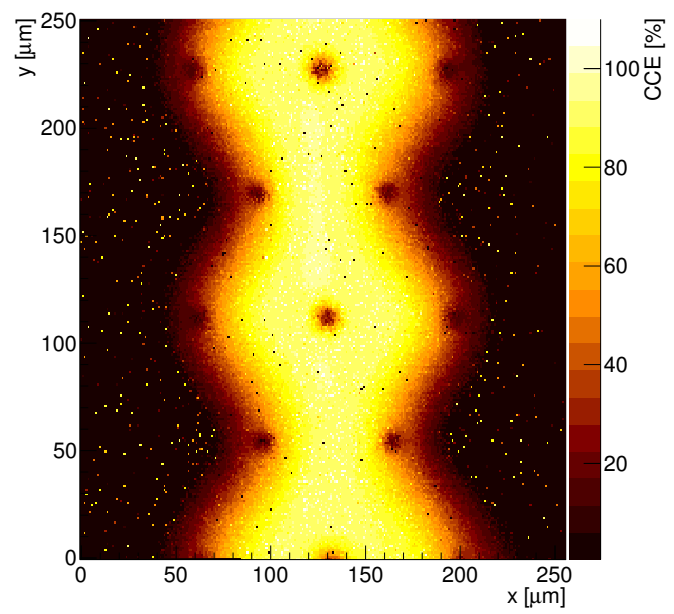

(c)

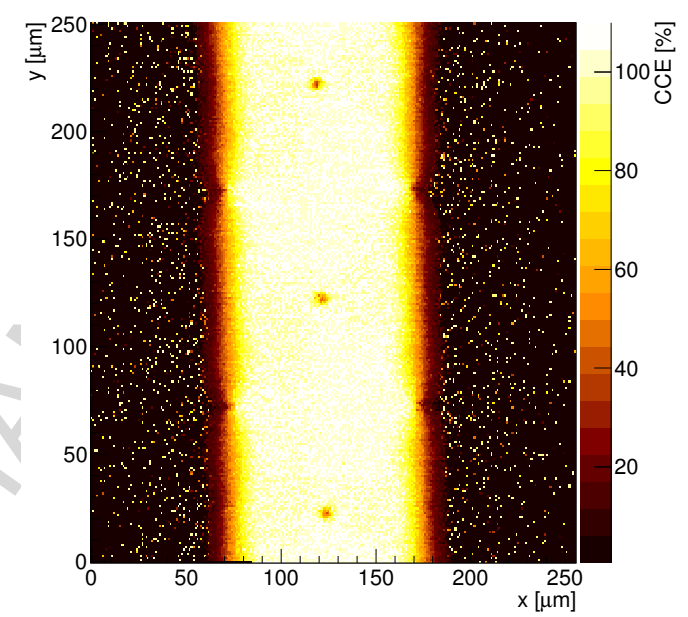

(b)

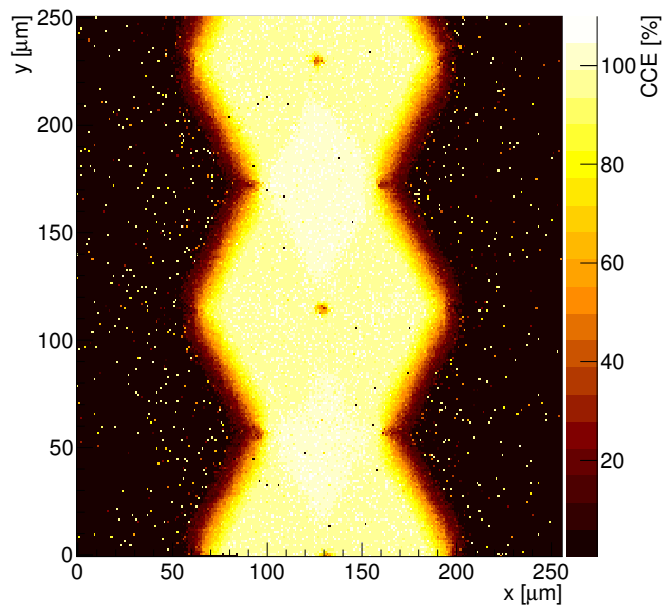

(d)

Figure 3: Charge collection efficiency (CCE) as a function of position measured with a proton micro-beam for square and hexagonal cells at a bias voltage of $-2 \mathrm{~V}$ and $-20 \mathrm{~V}$. (a) Square cells at $-2 \mathrm{~V}$. (b) Square cells at $-20 \mathrm{~V}$. (c) Hexagonal cells at $-2 \mathrm{~V}$. (d) Hexagonal cells at $-20 \mathrm{~V}$. 
Study of cubic and hexagonal cell geometries of a 3D diamond detector with a proton micro-beam - April $2017 \bullet$ Diamond and Related Materials

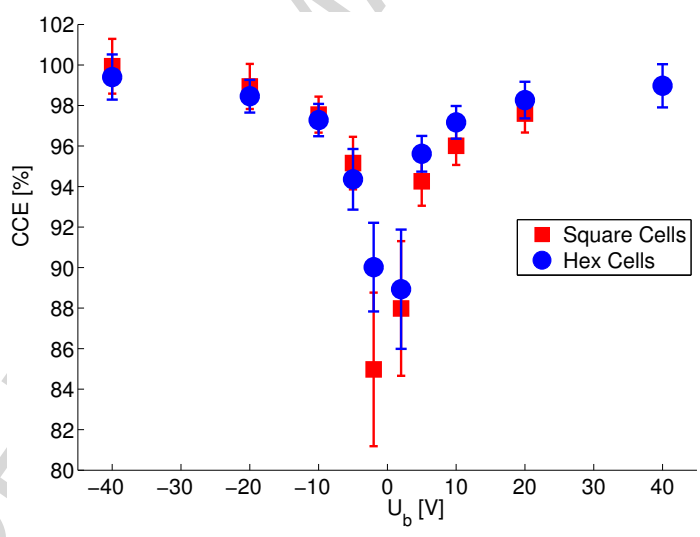

Figure 4: Average charge collection efficiency (CCE) as a function of bias voltage for square (red) and hexagonal (blue) cells. 
Study of cubic and hexagonal cell geometries of a 3D diamond detector with a proton micro-beam - April 2017 • Diamond and Related Materials

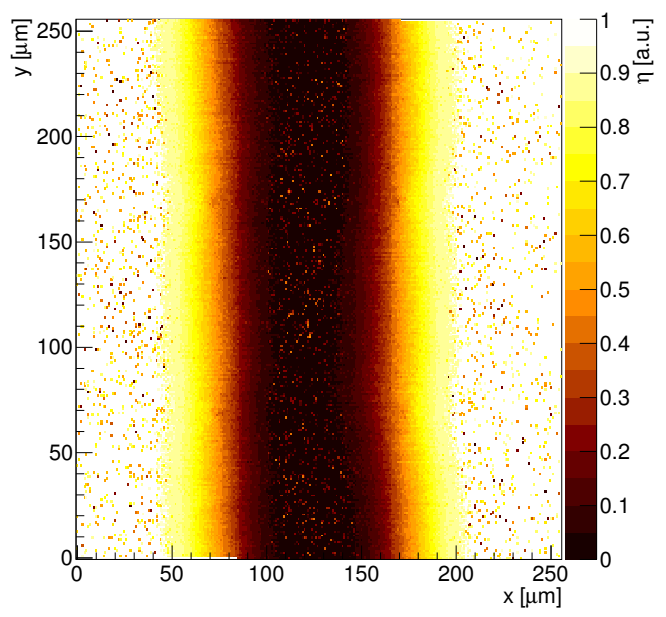

(a)

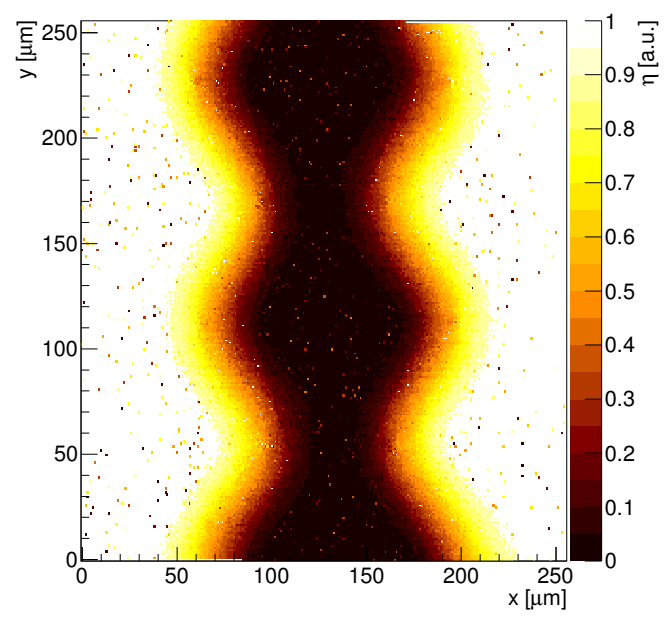

(c)

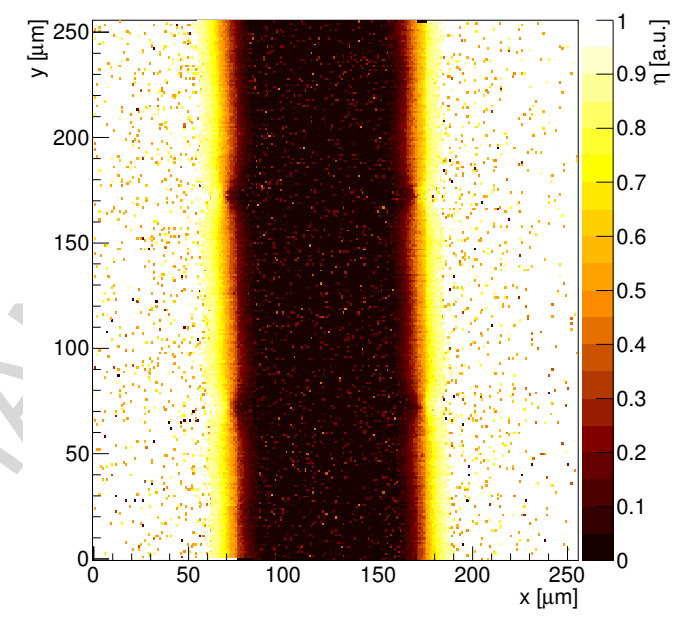

(b)

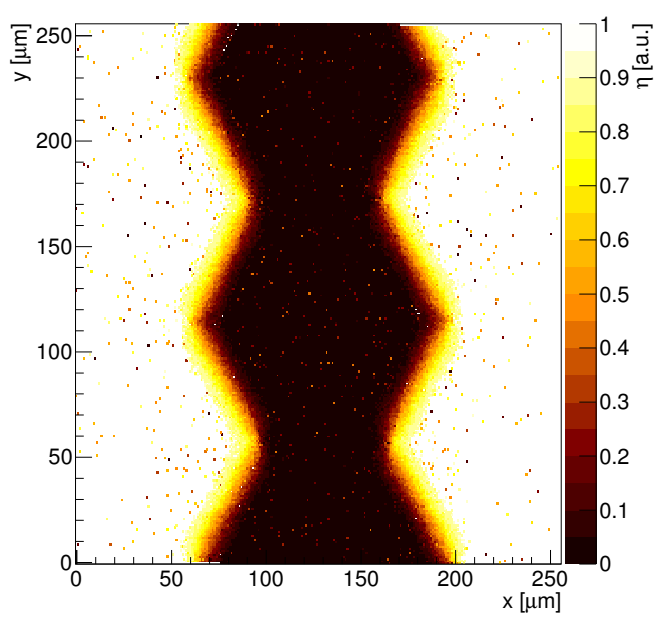

(d)

Figure 5: Regions of charge sharing between adjacent readout lines for square and hexagonal cells at $-2 \mathrm{~V}$ and $-20 \mathrm{~V}$. (a) Square cells at -2 V. (b) Square cells at $-20 \mathrm{~V}$. (c) Hexagonal cells at -2 V. (d) Hexagonal cells at $-20 \mathrm{~V}$. 
Study of cubic and hexagonal cell geometries of a 3D diamond detector with a proton micro-beam - April 2017 • Diamond and Related Materials

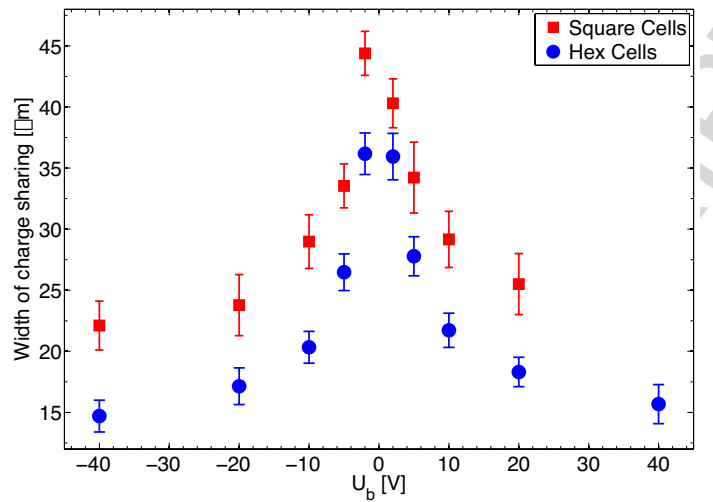

Figure 6: The width of the charge sharing regions as a function of bias voltage for square (red) and hexagonal (blue) cells.

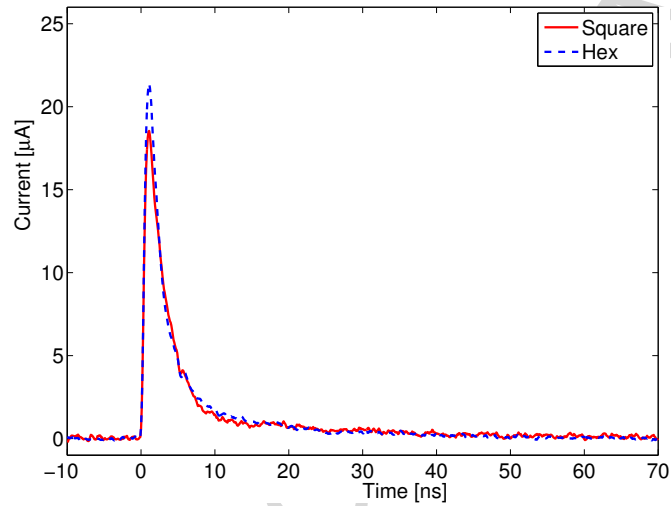

(a)

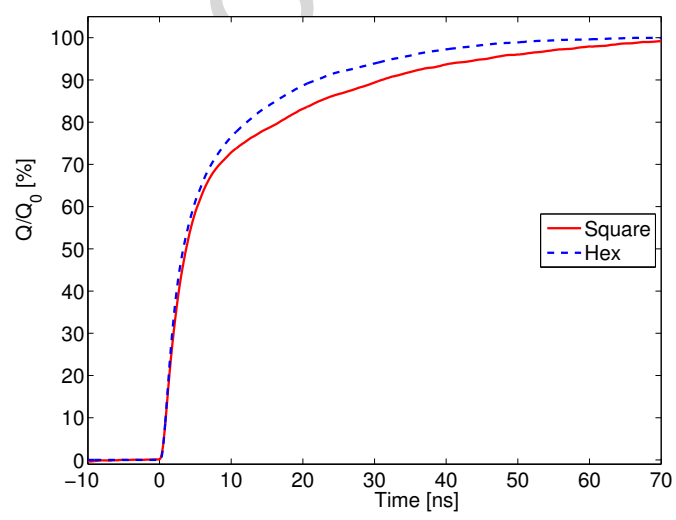

(c)

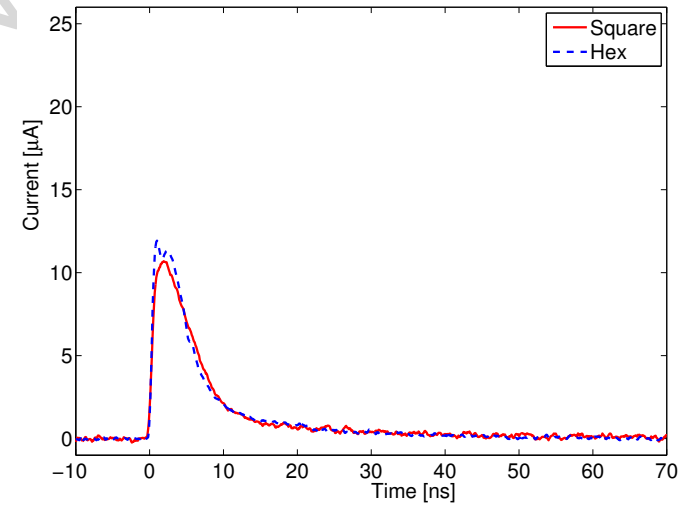

(b)

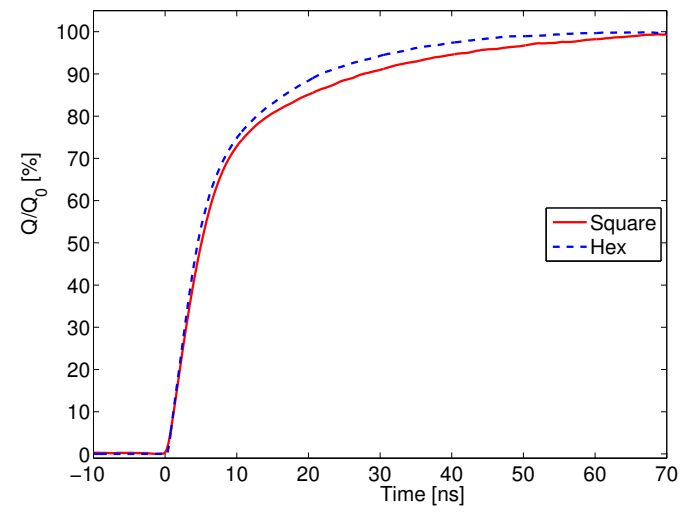

(d)

Figure 7: Transient current signal from TRIBIC data in (a) a high field region, and ( $b$ ) a low field region at a bias voltage of $-20 \mathrm{~V}$. Cumulative integral of the current pulses are shown in (c) and (d). 
Study of cubic and hexagonal cell geometries of a 3D diamond detector with a proton micro-beam - April 2017 • Diamond and Related Materials

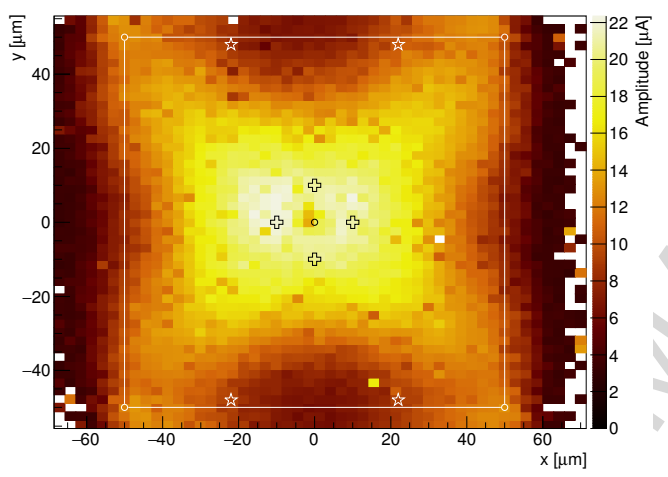

(a)

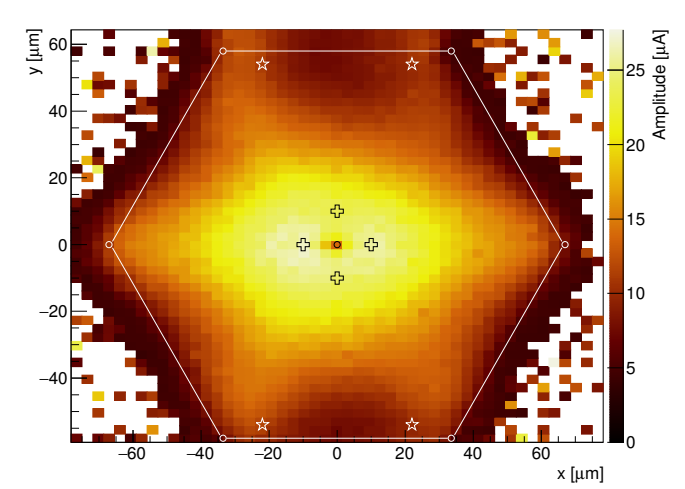

(c)

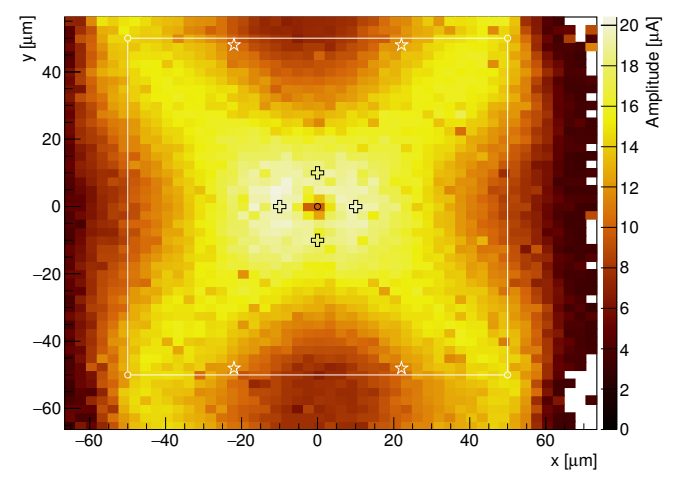

(b)

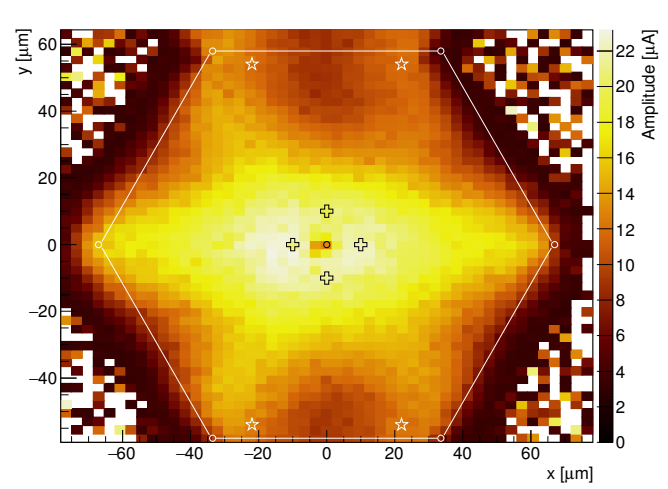

(d)

Figure 8: Amplitude plots of transient current signals from TRIBIC data at a bias voltage of $\pm 20 \mathrm{~V}$. (a) Square cell at $+20 \mathrm{~V}$. (b) Square cell at $-20 \mathrm{~V}$. (c) Hexagonal cell at $+20 \mathrm{~V}$. (d) Hexagonal cell at $-20 \mathrm{~V}$. The cell boundary is indicated by the solid lines, the electrodes by the open circles, and additionally the low field (open crosses) and high field (open stars) regions referring to Figure 7 are marked. 
Study of cubic and hexagonal cell geometries of a 3D diamond detector with a proton micro-beam - April 2017 • Diamond and Related Materials

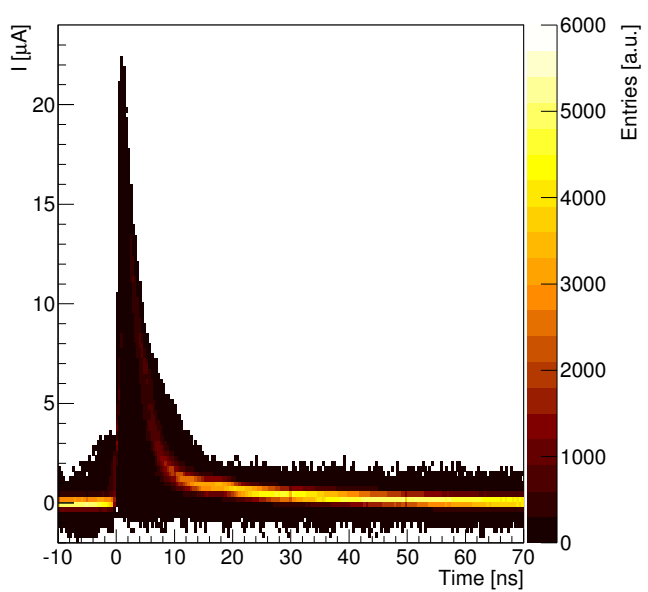

(a)

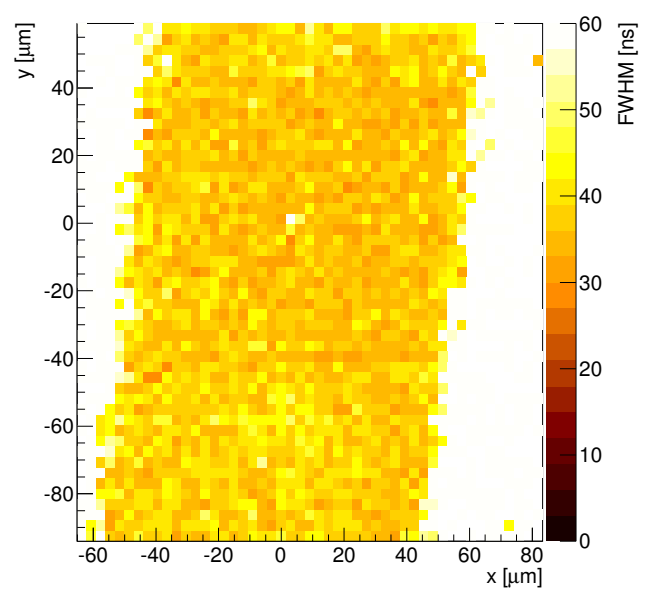

(c)

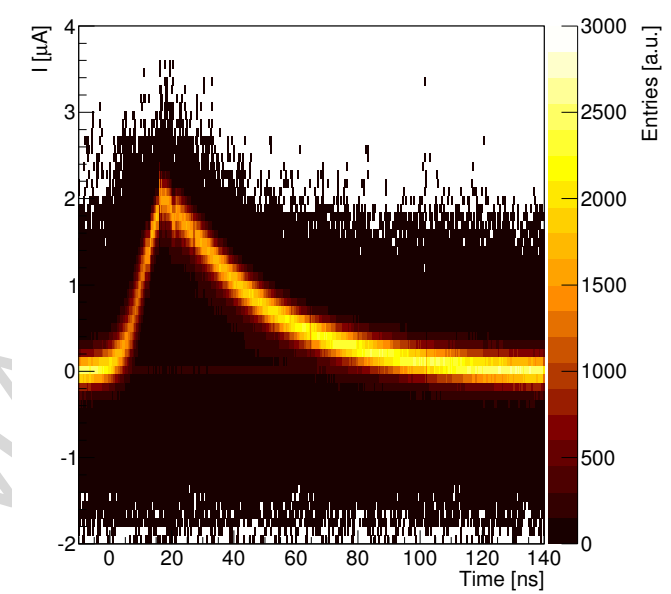

(b)

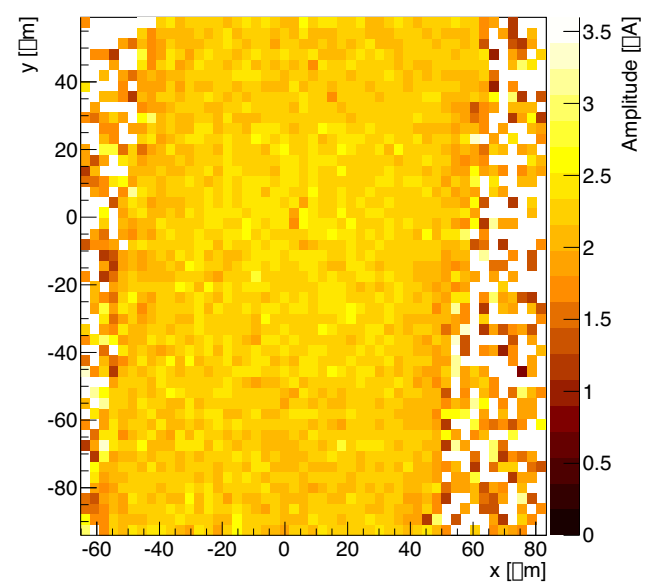

(d)

Figure 9: A density plot of transient current signals from TRIBIC data for protons incident on (a) the readout side and (b) the bias side of the detector. A 2D plot of the FWHM (c) and maximum amplitude (d) for protons incident on the bias side taken at a bias voltage of $+20 \mathrm{~V}$. Note that the tilt has not been corrected in (c) and (d). 
Study of cubic and hexagonal cell geometries of a 3D diamond detector with a proton micro-beam - April 2017 • Diamond and Related Materials

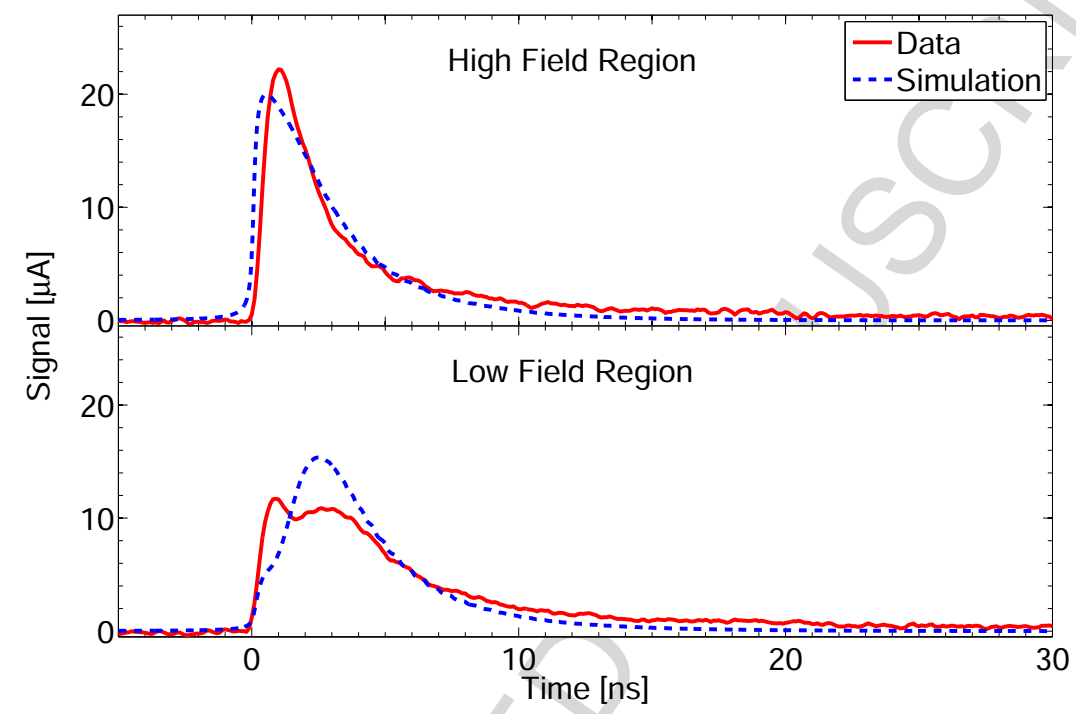

(a)

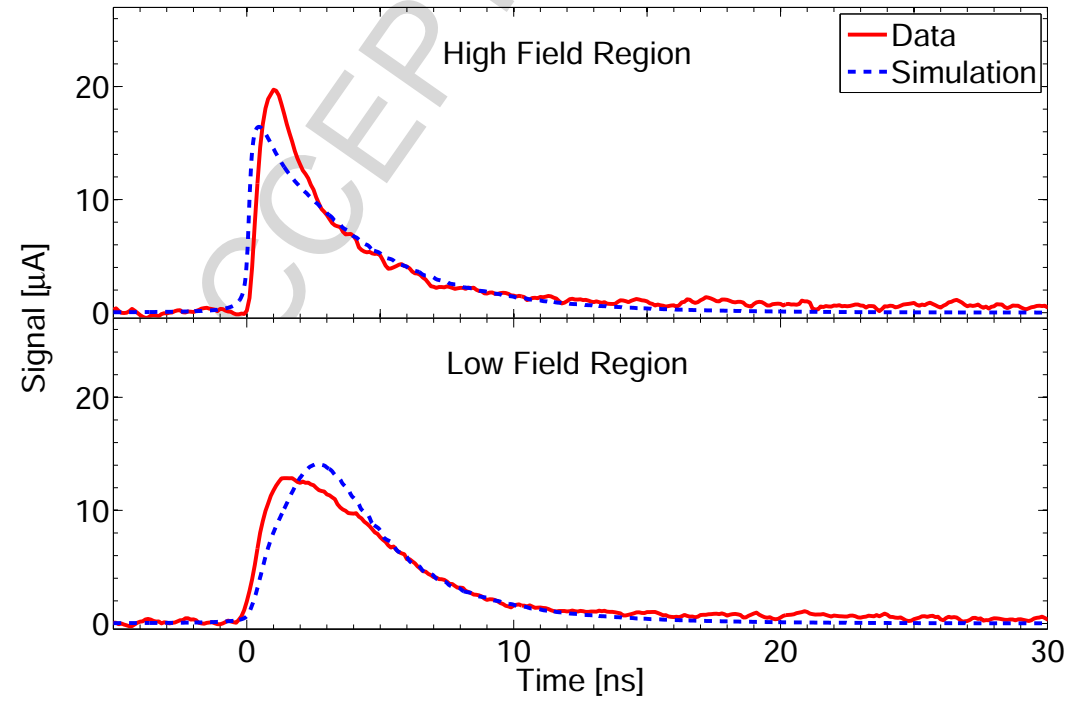

(b)

Figure 10: Comparison of simulation and data of a TRIBIC signal for the hexagonal (a) and cubic (b) cells in the low high field regions, the proton beam impinging on the read-out side. The results are shown for a bias voltage of -20V. 
Study of cubic and hexagonal cell geometries of a 3D diamond detector with a proton micro-beam - April 2017 • Diamond and Related Materials

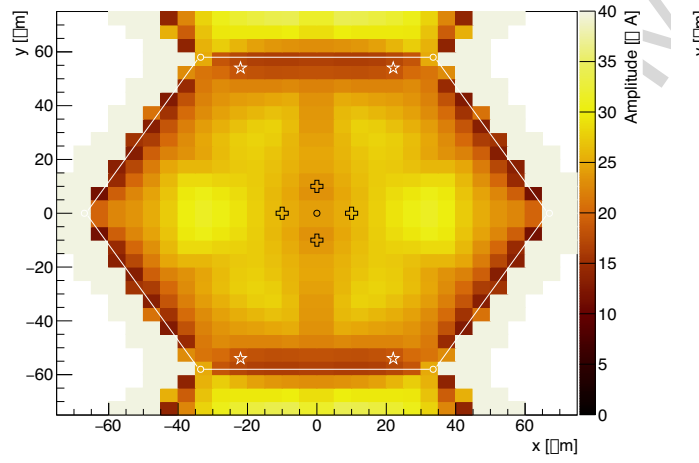

(a)

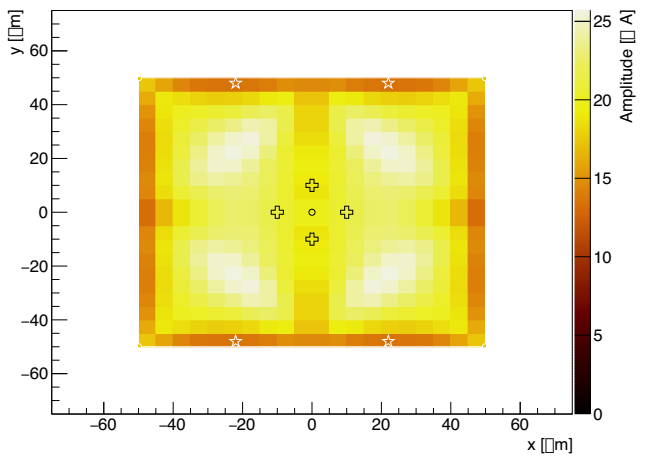

(b)

Figure 11: Amplitude plots from a simulation of the TRIBIC signal for the hexagonal (a) and cubic (b) cells at a bias voltage of $+20 \mathrm{~V}$ and the proton beam impinging on the read-out side. 
Study of cubic and hexagonal cell geometries of a 3D diamond detector with a proton micro-beam - April 2017 • Diamond and Related Materials

\section{GRAPHICAL ABSTRACT}

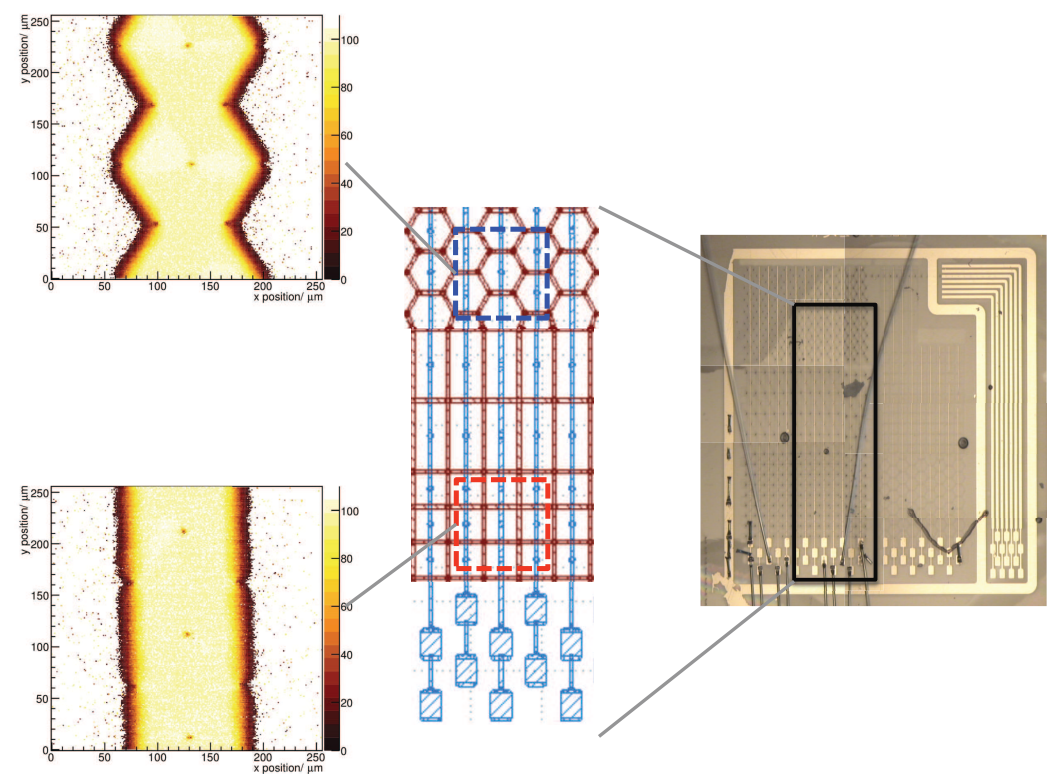


Study of cubic and hexagonal cell geometries of a 3D diamond detector with a proton micro-beam - April 2017 • Diamond and Related Materials

\section{Highlights}

- 3D diamond detector with hexagonal and cubic cells fabricated.

- Performance of hexagonal and cubic cells comparable.

- Differences in charge sharing observed, important for application scenario. 